\title{
Statistical cluster point and statistical limit point sets of subsequences of a given sequence
}

\author{
Harry I. Miller, Leila Miller-Van Wieren*(D) \\ Faculty of Engineering and Natural Sciences, International University of Sarajevo, Sarajevo, 71000, \\ Bosnia-Herzegovina
}

\begin{abstract}
J.A. Fridy [Statistical limit points, Proc. Amer. Math. Soc., 1993] considered statistical cluster points and statistical limit points of a given sequence $x$. Here we show that almost all subsequences of $x$ have the same statistical cluster point set as $x$. Also, we show an analogous result for the statistical limit points of $x$.
\end{abstract}

Mathematics Subject Classification (2010). 40D25, 40G99, 28A12

Keywords. sequences, subsequences, statistical cluster points, statistical limit points

\section{Introduction}

Fridy [1] has proven that $\Gamma_{x}$, the set of statistical cluster points of $x=\left(x_{n}\right)$, is always a closed set and $\Gamma_{x}$ is non-empty if $x$ is bounded. However $\Lambda_{x}$, the set of statistical limit points of $x$, need not be closed. In [2] H.I. Miller studied statistical convergence and relations between statistical convergence of a sequence $x$ and statistical convergence of the subsequences of $x$. In particular, in [2], it is shown that if $L$ is the statistical limit of $x$, then almost all subsequences of $x$ have $L$ as their statistical limit. Here we combine two notions, statistical cluster points and subsequences, showing that $\Gamma_{x}$ is equal to the statistical cluster point set of almost all subsequences of $x$. This is a continuation of the results in [3] that also combine statistical cluster points and subsequences. Namely, in [3] it is shown that if $\Gamma_{x} \neq \emptyset$ and $F$ is a non-empty closed subset of $\Gamma_{x}$, then there exists a subsequence $y$ of $x$ such that $\Gamma_{y}=F$. Additionally we show that $\Lambda_{x}$ is equal to the statistical limit point set of almost all subsequences of $x$. This is a continuation of the results in [4] that also combine statistical limit points and subsequences.

\section{Preliminaries}

If $t \in(0,1]$, then $t$ has a unique binary expansion $t=\sum_{n=1}^{\infty} \frac{e_{n}}{2^{n}}, e_{n} \in\{0,1\}$, with infinitely many ones. Next if $x=\left(x_{n}\right)$ is a sequence of reals, for each $t \in(0,1]$, let $x(t)$ denote the subsequence of $x$ obtained by the following rule: $x_{n}$ is in the subsequence if and only if $e_{n}=1$. Clearly the mapping $t \rightarrow x(t)$ is a one-to-one onto mapping between $(0,1]$ and the collection of all subsequences of $x$.

\footnotetext{
*Corresponding Author.

Email addresses: himiller@hotmail.com (H.I. Miller), lmiller@ius.edu.ba (L.M. Wieren)

Received: 10.07.2016; Accepted: 06.10.2016
} 
If $K$ is a subset of the positive integers $N$, then following Fridy [1], $K_{n}$ denotes the set $\{k \in K: k \leq n\}$ and $\left|K_{n}\right|$ denotes the number of elements in $K_{n}$. The natural density of $K$ (see [5]) is given by $\delta(K)=\lim _{n \rightarrow \infty} n^{-1}\left|K_{n}\right|$, provided this limit exists. In the case that $\delta(K)=0$ we say that $K$ is thin, and otherwise we say that $K$ is non-thin.

Statistical convergence of a sequence is defined as follows.

We say that $L$ is the statistical limit of the sequence $x$, if for every $\epsilon>0$,

$$
\lim _{n \rightarrow \infty} \frac{1}{n}\left|\left\{k \leq n:\left|x_{k}-L\right| \geq \epsilon\right\}\right|=0 .
$$

Statistical convergence and its connection to subsequences is studied in [2].

Statistical limit points and statistical cluster points of a sequence $x$ are defined as follows.

We say that a number $\lambda$ is a statistical limit point of a sequence of reals $x=\left(x_{n}\right)$ if $\lim _{k \rightarrow \infty} x_{n_{k}}=\lambda$ for some non-thin subsequence of $\left(x_{n}\right)$.

We say that a number $\gamma$ is a statistical cluster point of a sequence of reals $\left(x_{n}\right)$ if for every $\epsilon>0$ the set $\left\{k \in N:\left|x_{k}-\gamma\right|<\epsilon\right\}$ is non-thin.

In [1], given a sequence $x$, three sets are considered. $L_{x}$, the set of limit points of $x$; $\Lambda_{x}$, the set of statistical limit points of $x$, and $\Gamma_{x}$, the set of statistical cluster points of $x$. Also, if $x$ is bounded, then $\Gamma_{x}$ is closed and non-empty.

In this paper we want to examine, $\Gamma_{x}$ and its relation to $\Gamma_{x(t)}$. Additionally we also consider $\Lambda_{x}$ and its relation to $\Lambda_{x(t)}$.

\section{Results}

Our main result is the following.

Theorem 3.1. If $x=\left(x_{n}\right)$ is a bounded sequence, then $\Gamma_{x}=\Gamma_{x(t)}$ for almost all $t \in(0,1]$ (in the sense of Lebesgue measure).

Proof. Since $\Gamma_{x}$ is closed, it is either finite or separable, i.e. there is a countable subset of $\Gamma_{x},\left\{l_{n}: n \in N\right\}$ such that its closure is $\Gamma_{x}$. We consider only the second case, the proof in the first case is much simpler.

First we show that $\Gamma_{x} \subseteq \Gamma_{x(t)}$ for almost all $t$. It is sufficient to show that $m\left(B_{n}\right)=1$ for $n=1,2, \ldots$ where $B_{n}=\left\{t \in(0,1]: l_{n} \in \Gamma_{x(t)}\right\}$. This is true since in that case $m(B)=1$ for $B=\bigcap_{n=1}^{\infty} B_{n}$ and then $\left\{l_{n}: n \in N\right\} \subseteq \Gamma_{x(t)}$ for all $t \in B$ and consequently $\Gamma_{x} \subseteq \Gamma_{x(t)}$ for all $t \in B$.

Since $l_{n} \in \Gamma_{x}$, then for every $\epsilon>0,\left\{k \in N:\left|x_{k}-l_{n}\right|<\epsilon\right\}$ is non-thin. If $\epsilon=\frac{1}{j}$ we can denote the above set by $\left\{k_{1}^{j}, k_{2}^{j}, k_{3}^{j}, \ldots\right\}$. Then, since it is non-thin there exists $\delta_{j}>0$ such that

$$
\frac{1}{p}\left|\left\{i: k_{i}^{j} \leq p\right\}\right|>\delta_{j}
$$

for infinitely many $p$. We can assume that $p=k_{M}^{j}$ for infinitely many sufficiently large $M$. Now for each $j$, by the Law of Large Numbers, the limiting frequency of $x_{k_{i}^{j}} i=$ $1,2, \ldots$ among the sequence $x(t)$ is $\frac{1}{2}$ for almost all $t \in(0,1]$, i.e. if $t=\sum_{m=1}^{\infty} \frac{e_{m}}{2^{m}}$, then $\lim _{m \rightarrow \infty} \frac{1}{m} \sum_{i=1}^{m} t_{k_{i}}^{j}=\frac{1}{2}$ for almost all $t \in(0,1]$. That is, $m\left(D_{j}\right)=1$, where

$$
D_{j}=\left\{t \in(0,1]: \lim _{m \rightarrow \infty} \frac{1}{m} \sum_{i=1}^{m} t_{k_{i}}^{j}=\frac{1}{2}\right\}
$$

for all $j$. Hence if $D=\bigcap_{j=1}^{\infty} D_{j}, m(D)=1$. Now we will check that $l_{n}$ is a statistical cluster point for each $t$ in $D$.

To see this we will show that $\left\{i \in N:\left|x(t)_{i}-l_{n}\right|<\frac{1}{j}\right\}$ is non-thin for every $j \in N$ and every $t \in D_{j}$. 
Consider the earlier mentioned $p=k_{M}^{j}$ for $M$ large enough. Then the number of such $i \leq p$, with $\left|x_{i}-l_{n}\right|<\frac{1}{j}$ is greater than $p \delta_{j}$. Now take $t \in D_{j}$. By (3.1), $\lim _{m \rightarrow \infty} \frac{1}{m} \sum_{i=1}^{m} t_{k_{i}}^{j}=\frac{1}{2}$. So for large $M, p=k_{M}^{j}$, we have

$$
\frac{1}{p}\left|\left\{i \leq p:\left|x(t)_{i}-l_{n}\right|<\frac{1}{j}\right\}\right|>\frac{\delta_{j}}{4},
$$

i.e. this holds for infinitely many $p$, i.e. $\left\{i \in N:\left|x(t)_{i}-l_{n}\right|<\frac{1}{j}\right\}$ is non-thin for every $j \in N$ and every $t \in D_{j}$. Hence $l_{n}$ is a statistical cluster point for every $t \in D$. This completes the proof that $\Gamma_{x} \subseteq \Gamma_{x(t)}$ for almost all $t$.

Next we show that $\Gamma_{x(t)} \subseteq \Gamma_{x}$ for almost all $t$. We will show that this inclusion holds for all normal $t \in(0,1]$, i.e. for all $t=\sum_{n=1}^{\infty} \frac{e_{n}}{2^{n}}$ for which $\lim _{n \rightarrow \infty} \frac{1}{n} \sum_{i=1}^{n} e_{i}=\frac{1}{2}$. It is well known that almost all $t \in(0,1]$ are normal (see [5] ).

Suppose that $l$ is a statistical cluster point of $x(t)$ for some normal $t$. Then for any $\epsilon>0,\left\{i:\left|(x(t))_{i}-l\right|<\epsilon\right\}$ is non-thin, i.e. there exists $\delta_{\epsilon}>0$ such that

$$
\frac{1}{n}|i \leq n:|(x(t))_{i}-l|<\epsilon|>2 \delta_{\epsilon}
$$

for infinitely many $n$. This implies that

$$
\frac{1}{n}|i \leq n:| x_{i}-l|<\epsilon|>\frac{1}{2} \delta_{\epsilon}
$$

for infinitely many $n$, and hence $l$ is a statistical cluster point of $x$. Therefore $\Gamma_{x(t)} \subseteq \Gamma_{x}$ for all normal $t$, and consequently for almost all $t \in(0,1]$. Therefore we conclude that $\Gamma_{x(t)}=\Gamma_{x}$ for almost all $t \in(0,1]$.

Next, we will prove an analogous result for the set of statistical limit points of $x$ and its subsequences. The set $\Lambda_{x}$ is not necessarily closed (see [4]). However the following useful theorem was proved by Kostyrko, Mačaj, Šalat and Strauch [4].

Theorem 3.2. For every bounded sequence $x$, the set $\Lambda_{x}$ is an $F_{\sigma}$-set in $R$.

In the proof of the above theorem, the authors show that

$$
\Lambda_{x}=\bigcup_{j=1}^{\infty} \Lambda\left(x, \frac{1}{j}\right)
$$

where $\Lambda\left(x, \frac{1}{j}\right)=\left\{l, \exists k_{i}, i=1,2 \ldots, \lim _{i \rightarrow \infty} x_{k_{i}}=l, \bar{\delta}\left(\left\{k_{i}\right\}\right) \geq \frac{1}{j}\right\}$ where $\bar{\delta}$ denotes the upper statistical density (i.e. $\left.\bar{\delta}\left(\left\{k_{i}\right\}\right)=\lim \sup _{i \rightarrow \infty} \frac{i}{k_{i}}\right)$ and $\Lambda\left(x, \frac{1}{j}\right)$ is closed for all $j$.

Here is our second result.

Theorem 3.3. If $x=\left(x_{n}\right)$ is a bounded sequence, then $\Lambda_{x}=\Lambda_{x(t)}$ for almost all $t \in(0,1]$ (in the sense of Lebesgue measure).

Proof. We proceed in a similar manner as in the proof of Theorem 3.1.

First we show that $\Lambda_{x} \subseteq \Lambda_{x(t)}$ for almost all $t$.

As mentioned earlier, $\Lambda_{x}=\bigcup_{j=1}^{\infty} T_{j}$, where

$$
T_{j}=\Lambda\left(x, \frac{1}{j}\right)=\left\{l, \exists k_{i}, i=1,2 \ldots, \lim _{i \rightarrow \infty} x_{k_{i}}=l, \bar{\delta}\left(\left\{k_{i}\right\}\right) \geq \frac{1}{j}\right\} .
$$

Suppose $j \in N$ is fixed. Using the above notation (from [4]), $T_{j}$ is closed and separable so there exists a set $\left\{l_{i j}: i \in N\right\}$ such that its closure is $T_{j}$. Let $i \in N$. If $l=l_{i j}$, then by the Law of Large Numbers, $l \in \Lambda\left(x(t), \frac{1}{4 j}\right)$, for all $t \in B_{i j}$, where $m\left(B_{i j}\right)=1$. Let $B_{j}=\bigcap_{i=1}^{\infty} B_{i j}$. Then $m\left(B_{j}\right)=1$. Hence $\left\{l_{i j}: i \in N\right\} \subseteq \Lambda\left(x(t), \frac{1}{4 j}\right)$ for every $t \in B_{j}$. Now since $T_{j}$ and $\Lambda\left(x(t), \frac{1}{4 j}\right)$ are both closed we get that $T_{j} \subseteq \Lambda\left(x(t), \frac{1}{4 j}\right)$ for every $t \in B_{j}$. 
Therefore $\Lambda_{x}=\bigcup_{j=1}^{\infty} T_{j} \subseteq \bigcup_{j=1}^{\infty} \Lambda\left(x(t), \frac{1}{4 j}\right)=\Lambda_{x(t)}$ for all $t \in \bigcap_{j=1}^{\infty} B_{j}$. Since $m\left(\bigcap_{j=1}^{\infty} B_{j}\right)=1$, we have shown that $\Lambda_{x} \subseteq \Lambda_{x(t)}$ for almost all $t$.

Next we show that $\Lambda_{x(t)} \subseteq \Lambda_{x}$ for almost all $t$. Again we show that this inclusion holds for all normal $t \in(0,1]$. Suppose that $l$ is a statistical limit point of $x(t)$ for some normal $t$. Then $x(t)$ has a non-thin subsequence that converges to $l$ (in the normal sense). It is easy to see that this subsequence $x(t)_{i}=x_{k_{i}}$ is then also a non-thin subsequence of $x$ and therefore $l$ is also a statistical limit point of $x$. This completes the proof.

\section{Concluding remarks}

We mentioned that $m(\nu)=1$, where $\nu$ is the set of normal numbers in $(0,1]$. However $\nu$ is a set of first Baire category. In light of this we suspect that a category analogue of our Theorem 3.1 is not true.

Also, one could examine possible analogues of our results using permutations rather than subsequences.

\section{References}

[1] J.A. Fridy, Statistical limit points, Proc. Amer. Math. Soc. 118 1187-1192, 1993.

[2] H.I. Miller, Measure theoretical subsequence characterization of statistical convergence, Trans. Amer. Math. Soc. 347 (5) 1811-1819, 1995.

[3] H.I. Miller and L. Miller-Van Wieren, Some statistical cluster point theorems, Hacet. J. Math. Stat. 44 (6) 1405-1409, 2015.

[4] P. Kostyrko, M. Mačaj, T. Šalat, and O. Strauch, On statistical limit points, Proc. Amer. Math. Soc. 129 (9), 2647-2654, 2000.

[5] J.C. Oxtoby, Measure and Category: A survey of the analogies between topological and measure spaces. Second edition, Springer-Verlag, New York-Berlin,1980. 